\title{
CURVATURE PINCHING FOR KAEHLER SUBMANIFOLDS OF A COMPLEX PROJECTIVE SPACE*
}

\author{
By \\ Yoshio MatsuYAMA
}

\begin{abstract}
A complete classification for a compact Kaehler submanifold $M_{n}$ in $P_{n+p}(C)$ with the scalar curvature $\rho \geq n^{2}$ is given, so that a conjecture of $\mathrm{K}$. Ogiue is resolved partially.
\end{abstract}

\section{Introduction}

Let $P_{n+p}(C)$ be an $(n+p)$-dimensional complex projective space with the Fubini-Study metric of constant holomorphic sectional curvature 1 . There are a number of conjectures for Kaehler submanifolds in $P_{n+p}(C)$ suggested by $\mathrm{K}$. Ogiue ([8]); some have been resolved under a suitable topological restriction (e.g. $M_{n}$ is complete) (cf. [1], [2], [8], [9], [10], [11], [12], [13], [14], [16] and [17]). In this direction, one of the open problems so far is as follows:

Conjecture (K. Ogiue). Let $M_{n}$ be an $n$-dimensional complete submanifold immersed in $P_{n+p}(C)$. If $\rho>n^{2}$, is $M$ totally geodesic in $P_{n+p}(C)$ ?

In the case that $M_{n}$ is a complete Kaehler submanifold immersed in $P_{n+p}(C)$ which has the Ricci curvature $S>\frac{n}{2}$, it was proved in [9] that such a submanifold $M_{n}$ is totally geodesic in $P_{n+p}(C)$ ([9]). Recently, in the case of $M_{n}$ has $S \geq \frac{n}{2}$ Suh and Yang ([12]) proved that such one is parallel, i.e., either totally geodesic or congruent to one of $Q_{n}$ and $P_{1}(C) \times P_{1}(C)$. Also, the case that the scalar curvature $\rho>n(n+1)-\frac{n+2}{3}$ was studied by Tanno [15], and he proved that $M$ is totally geodesic in $P_{n+p}(C)$.

* 2000 Mathematics Subject Classification. Primary 53C40; Secondary 53B25.

Key words and phrases. Complex projective space, complex Kaehler submanifold, parallel second fundamental form.

Received October 18, 2007. 
In the present paper we would like to consider the case that $M_{n}$ is compact and $\rho>n^{2}$, so that the above conjecture is resolved partially. The main result is the following:

THEOREM. Let $M_{n}$ be an $n$-dimensional compact Kaehler submanifold immersed in $P_{n+p}(C)$. Then $\rho \geq n^{2}$ if and only if $M$ is either totally geodesic in $P_{n+p}(C)$ or $\rho=n^{2}$. In the latter case $M^{n}$ is imbedded submanifold congruent to the standard imbedding of one of the following submanifolds: $P_{1}(C) \times P_{1}(C)$ and the complex quadric $Q_{n}, n \geq 3$.

Hence, we have the following (see [8], p662-p663):

COROLlARY. Let $M_{n}$ be an n-dimensional compact Kaehler submanifold immersed in $P_{n+p}(C)$. If $\rho>n^{2}$, then $M$ is totally geodesic in $P_{n+p}(C)$.

\section{Preliminaries}

Let $M_{n}$ be a compact Kaehler submanifold of complex dimension $n$, immersed in the complex projective space $P_{n+p}(C)$ endowed with the Fubini-Study metric of constant holomorphic sectional curvature 1 . We denote by $U M$ the unit tangent bundle over $M$ and by $U M_{x}$ its fibre over $x \in M$ and by $J$ and $\langle$,$\rangle the complex$ structure and the Fubini-Study metric. Let $\nabla$ and $h$ be the Riemannian connection and the second fundamental form of the immersion, respectively. $A$ and $\nabla^{\perp}$ are the Weingarten endomorphism and the normal connection. The first and the second covariant derivatives of the normal valued tensor $h$ are given by

$$
(\nabla h)(X, Y, Z)=\nabla_{X}^{\perp}(h(Y, Z))-h\left(\nabla_{X} Y, Z\right)-h\left(Y, \nabla_{X} Z\right)
$$

and

$$
\begin{aligned}
\left(\nabla^{2} h\right)(X, Y, Z, W)= & \nabla_{X}^{\perp}((\nabla h)(Y, Z, W))-(\nabla h)\left(\nabla_{X} Y, Z, W\right) \\
& -(\nabla h)\left(Y, \nabla_{X} Z, W\right)-(\nabla h)\left(Y, Z, \nabla_{X} W\right),
\end{aligned}
$$

respectively, for any vector fields $X, Y, Z$ and $W$ tangent to $M_{n}$.

Let $R$ and $R^{\perp}$ denote the curvature tensor associated with $\nabla$ and $\nabla^{\perp}$, respectively. Then $h$ and $\nabla h$ are symmetric and for $\nabla^{2} h$ we have the Ricci-identity

$$
\begin{aligned}
& \left(\nabla^{2} h\right)(X, Y, Z, W)-\left(\nabla^{2} h\right)(Y, X, Z, W) \\
& \quad=R^{\perp}(X, Y) h(Z, W)-h(R(X, Y) Z, W)-h(Z, R(X, Y) W) .
\end{aligned}
$$


We also consider the relations

$$
h(J X, Y)=J h(X, Y) \text { and } A_{J \xi}=J A_{\xi}=-A_{\xi} J,
$$

where $\xi$ is a normal vector to $M_{n}$.

If $S$ and $\rho$ is the Ricci tensor of $M$ and the scalar curvature of $M$, respectively, since $M$ is a complex Kaehler submanifold in $P_{n+p}(C)$, then from the Gauss equation we have

$$
\begin{aligned}
S(v, w) & =\frac{n+1}{2}\langle v, w\rangle-\sum_{i=1}^{2 n}\left\langle A_{h\left(v, e_{i}\right)} e_{i}, w\right\rangle, \\
\rho & =n(n+1)-|h|^{2} .
\end{aligned}
$$

Now, let $v \in U M_{x}, x \in M$. If $e_{2}, \ldots, e_{2 n}$ are orthonormal vectors in $U M_{x}$ orthogonal to $v$, then we can consider $\left\{e_{2}, \ldots, e_{2 n}\right\}$ as an orthonormal basis of $T_{v}\left(U M_{x}\right)$. We remark that $\left\{v=e_{1}, e_{2}, \ldots, e_{2 n}\right\}$ is an orthonormal basis of $T_{x} M$. We denote the Laplacian of $U M_{x} \cong S^{2 n-1}$ by $\Delta$.

Define a function $f_{1}$ on $U M_{x}, x \in M$, by

$$
f_{1}(v)=\sum_{i, j=1}^{2 n}\left\langle A_{h\left(e_{i}, e_{j}\right)} e_{j}, A_{h(v, v)} e_{i}\right\rangle .
$$

Noting that $\nabla_{e_{k}} v=-e_{k}, \nabla_{e_{k}} e_{\ell}=\delta_{k \ell} v, k, \ell=2, \ldots, 2 n$, we have

$$
\begin{aligned}
\left(\Delta f_{1}\right)(v) & =\sum_{k=2}^{2 n}\left(\nabla f_{1}\right)\left(v, e_{k}, e_{k}\right) \\
& =-2 \sum_{k=2}^{2 n} \nabla_{e_{k}}\left(\sum_{i, j=1}^{2 n}\left\langle A_{h\left(e_{i}, e_{j}\right)} e_{j}, A_{h\left(e_{k}, v\right)} e_{i}\right\rangle\right) \\
& =-2 \sum_{k=2}^{2 n} f_{1}(v)+2 \sum_{k=2}^{2 n} f_{1}\left(e_{k}\right) .
\end{aligned}
$$

Using the minimality of $M$ we can prove that

$$
\begin{aligned}
\left(\Delta f_{1}\right)(v) & =-2(2 n-1) f_{1}(v)+2 \sum_{k=2}^{2 n}\left\langle A_{h\left(e_{i}, e_{j}\right)} e_{j}, A_{h\left(e_{k}, e_{k}\right)} e_{i}\right\rangle \\
& =-4 n f_{1}(v) .
\end{aligned}
$$

For more details on this, see [7], [10]. Similarly, define $f_{2}, f_{3}, f_{4}, f_{5}, f_{6}, f_{7}, f_{8}, f_{9}$, $f_{10}$ and $f_{11}$ by 


$$
\begin{aligned}
f_{2}(v) & =\sum\left\langle A_{h(v, v)} v, A_{h\left(v, e_{i}\right)} e_{i}\right\rangle, \\
f_{3}(v) & =\sum\left\langle A_{h\left(e_{i}, e_{j}\right)} e_{j}, A_{h\left(v, e_{i}\right)} v\right\rangle, \\
f_{4}(v) & =\sum\left\langle A_{h\left(v, e_{i}\right)} e_{i}, A_{h\left(v, e_{j}\right)} e_{j}\right\rangle, \\
f_{5}(v) & =\sum\left\langle A_{h(v, v)} e_{i}, A_{h(v, v)} e_{i}\right\rangle, \\
f_{6}(v) & =\sum\left\langle A_{h\left(e_{j}, v\right)} e_{i}, A_{h\left(e_{j}, v\right)} e_{i}\right\rangle \\
f_{7}(v) & =|h(v, v)|^{2}, \\
f_{8}(v) & =\sum\left\langle A_{h\left(v, e_{i}\right)} e_{i}, v\right\rangle|h(v, v)|^{2}, \\
f_{9}(v) & =\left(\sum\left\langle A_{h\left(v, e_{i}\right)} e_{i}, v\right\rangle\right)^{2}, \\
f_{10}(v) & =\sum\left\langle A_{h\left(v, e_{i}\right)} e_{i}, v\right\rangle \\
f_{11}(v) & =|h|^{2}|h(v, v)|^{2},
\end{aligned}
$$

respectively. Then we obtain

$$
\begin{aligned}
& \left(\Delta f_{2}\right)(v)=-4(2 n+2) f_{2}(v)+4 f_{3}(v)+4 f_{4}(v)+2 f_{1}(v), \\
& \left(\Delta f_{3}\right)(v)=-4 n f_{3}(v)+2 \sum\left\langle A_{h\left(e_{j}, e_{i}\right)} e_{j}, A_{h\left(e_{k}, e_{i}\right)} e_{k}\right\rangle \\
& \left(\Delta f_{4}\right)(v)=-4 n f_{4}(v)+2 \sum\left\langle A_{h\left(e_{j}, e_{i}\right)} e_{j}, A_{h\left(e_{k}, e_{i}\right)} e_{k}\right\rangle \\
& \left(\Delta f_{5}\right)(v)=-4(2 n+2) f_{5}(v)+8 \sum\left\langle A_{h\left(e_{j}, v\right)} e_{i}, A_{h\left(e_{j}, v\right)} e_{i}\right\rangle, \\
& \left(\Delta f_{6}\right)(v)=-4 n f_{6}(v)+2 \sum\left\langle A_{h\left(e_{j}, e_{k}\right)} e_{i}, A_{h\left(e_{j}, e_{k}\right)} e_{i}\right\rangle, \\
& \left(\Delta f_{7}\right)(v)=-4(2 n+2) f_{7}(v)+8 \sum\left\langle A_{h\left(v, e_{i}\right)} e_{i}, v\right\rangle, \\
& \left(\Delta f_{8}\right)(v)=-6(2 n+4) f_{8}(v)+16 f_{2}(v)+2 f_{11}(v)+8 f_{9}(v), \\
& \left(\Delta f_{9}\right)(v)=-4(2 n+2) f_{9}(v)+8 f_{4}(v)+4|h|^{2} \sum\left\langle A_{h\left(v, e_{i}\right)} e_{i}, v\right\rangle, \\
& \left(\Delta f_{10}\right)(v)=-4 n f_{10}(v)+2|h|^{2}, \\
& \left(\Delta f_{11}\right)(v)=-4(2 n+2) f_{11}(v)+8|h|^{2} \sum\left\langle A_{h\left(v, e_{i}\right)} e_{i}, v\right\rangle .
\end{aligned}
$$


Since

$$
\begin{aligned}
\frac{1}{2} \sum\left(\nabla^{2} f_{7}\right)\left(e_{i}, e_{i}, v\right) & =\sum\left\langle\left(\nabla^{2} h\right)\left(e_{i}, e_{i}, v, v\right), h(v, v)\right\rangle \\
& =\sum\left\langle(\nabla h)\left(e_{i}, v, v\right),(\nabla h)\left(e_{i}, v, v\right)\right\rangle
\end{aligned}
$$

we have the following (See [3], [4], [5], [6] and [7]):

Lemma. Let $M$ be an n-dimensional complex Kaehler submanifold of $P_{n+p}(C)$. Then for $v \in U M_{x}$ we have

$$
\begin{aligned}
\frac{1}{2} \sum\left(\nabla^{2} f_{7}\right)\left(e_{i}, e_{i}, v\right)= & \sum\left|(\nabla h)\left(e_{i}, v, v\right)\right|^{2}+\frac{n+2}{2}|h(v, v)|^{2} \\
& +2 \sum\left\langle A_{h(v, v)} e_{i}, A_{h\left(e_{i}, v\right)} v\right\rangle \\
& -2 \sum\left\langle A_{h\left(v, e_{i}\right)} e_{i}, A_{h(v, v)} v\right\rangle \\
& -\sum\left\langle A_{h(v, v)} e_{i}, A_{h(v, v)} e_{i}\right\rangle .
\end{aligned}
$$

\section{Proof of Theorem}

From (2) we have

$$
\rho=n(n+1)-|h|^{2} .
$$

Thus we have only to prove Theorem under the assumption

$$
|h|^{2} \leq n \text {. }
$$

We see the following equation holds for $v \in U M_{x}, x \in M$.

$$
\sum\left\langle A_{h(J v, J v)} e_{i}, A_{h\left(e_{i}, J v\right)} J v\right\rangle=-\sum\left\langle A_{h(v, v)} e_{i}, A_{h\left(e_{i}, v\right)} v\right\rangle .
$$

From (14) and (16) we have

$$
\begin{aligned}
\frac{1}{4} \sum & \left(\nabla^{2} f_{7}\right)\left(e_{i}, e_{i}, v\right)+\frac{1}{4} \sum\left(\nabla^{2} f_{7}\right)\left(e_{i}, e_{i}, J v\right) \\
= & \sum\left|(\nabla h)\left(e_{i}, v, v\right)\right|^{2}+\frac{n+2}{2}|h(v, v)|^{2} \\
& -2 \sum\left\langle A_{h\left(v, e_{i}\right)} e_{i}, A_{h(v, v)} v\right\rangle-\sum\left\langle A_{h(v, v)} e_{i}, A_{h(v, v)} e_{i}\right\rangle .
\end{aligned}
$$


Now, we choose an orthonormal basis $\left\{v=e_{1}, e_{2}, \ldots, e_{n}\right\}$ such that the matrix $\sum_{\alpha=1}^{2 p} A_{\xi_{\alpha}}^{2}$ is diagonalized, where $\left\{\xi_{1}, \xi_{2}, \ldots, \xi_{2 p}\right\}$ is any orthonormal normal basis and $1 \leq \alpha \leq 2 p$. Then we have

$$
f_{2}(v)=f_{8}(v)
$$

In terms of (4), (5), (6), (10), (11), (13), (17) and (18) we have

$$
\begin{aligned}
\frac{1}{4} \sum & \left(\nabla^{2} f_{7}\right)\left(e_{i}, e_{i}, v\right)+\frac{1}{4} \sum\left(\nabla^{2} f_{7}\right)\left(e_{i}, e_{i}, J v\right) \\
& +\frac{1}{6 n(2 n+2)}\left(2\left(\Delta f_{2}\right)(v)+\frac{2}{n}\left(\Delta f_{3}\right)(v)-\frac{2}{n}\left(\Delta f_{4}\right)(v)+\frac{1}{n}\left(\Delta f_{1}\right)(v)\right. \\
& \left.-(2 n+2)\left(\Delta f_{8}\right)(v)-2\left(\Delta f_{9}\right)(v)+\left(\Delta f_{11}\right)(v)\right) \\
= & \sum\left|(\nabla h)\left(e_{i}, v, v\right)\right|^{2}+\frac{n+2}{2}|h(v, v)|^{2}-\frac{1}{n} f_{11}(v)-f_{5}(v) \\
\geq & \sum\left|(\nabla h)\left(e_{i}, v, v\right)\right|^{2}+\frac{n}{2}|h(v, v)|^{2}-f_{5}(v)
\end{aligned}
$$

noting that (15). On the other hand, in terms of (9) and (12) we have

$$
\begin{gathered}
\frac{n}{2}\left(\frac{1}{4(2 n+2)}\left(\Delta f_{7}\right)(v)+\frac{2}{4 n(2 n+2)}\left(\Delta f_{10}\right)(v)\right) \\
=-\frac{n}{2}|h(v, v)|^{2}+\frac{n}{2 n(2 n+2)}|h|^{2} .
\end{gathered}
$$

Also, we have from (7) and (8)

$$
\begin{aligned}
& -\frac{1}{4(2 n+2)}\left(\Delta f_{5}\right)(v)-\frac{2}{4 n(2 n+2)}\left(\Delta f_{6}\right)(v) \\
& \quad=f_{5}(v)-\frac{2}{2 n(2 n+2)} \sum\left\langle A_{h\left(e_{j}, e_{k}\right)} e_{i}, A_{h\left(e_{j}, e_{k}\right)} e_{i}\right\rangle \\
& \quad=f_{5}(v)-\frac{2}{2 n(2 n+2)} \sum_{\alpha, \beta=1}^{2 p}\left(\operatorname{trace} A_{\xi_{\alpha}} A_{\xi_{\beta}}\right)^{2} \\
& \quad \geq f_{5}(v)-\frac{1}{2 n(2 n+2)}|h|^{4} \\
& \quad \geq f_{5}(v)-\frac{n}{2 n(2 n+2)}|h|^{2}
\end{aligned}
$$


where we used $\sum\left(\operatorname{trace} A_{\xi_{\alpha}} A_{\xi_{\beta}}\right)^{2} \leq \frac{1}{2}|h|^{4} \quad$ (See [9], p. 88) and (15), where $\left\{\xi_{1}, \xi_{2}, \ldots, \xi_{2 p}\right\}$ is any orthonormal normal basis as above and $1 \leq \alpha, \beta \leq 2 p$. Summing up (17), (20) and (21) and using Hopf's lemma, we have

$$
\sum\left|(\nabla h)\left(e_{i}, v, v\right)\right|^{2}=0 .
$$

Thus we know that $M_{n}$ is parallel. This proves Theorem (See [8], p. 662-663).

\title{
References
}

[1] Liao, R., Scalar curvature of Kaehler submanifolds of a complex projective space, Kexue Tongbao (English Ed.) 32 (1987), pp. 865-869.

[2] Matsuyama, Y., On a 2-dimensional Einstein Kaehler submanifolds of a complex space form, Proc. Amer. Math. Soc. 95 (1985), pp. 595-603.

[3] Matsuyama, Y., On some pinchings of minimal submanifolds, Proceedings of the Workshop on Geometry and its applications in Honor of Morio Obata, November 1991, edited by Tadashi Nagano et al., World Scieentific, Singapore (1993), pp. 121-134.

[4] Matsuyama, Y., On curvature pinching for totally real submanifolds of $C P^{n}(c)$, J. Ramanujan Math. Soc. 9 (1994), pp 13-24.

[ 5 ] Matsuyama, Y., Curvature pinching for totally real submanifolds of a complex projective space, J. Math. Soc. Japan 52 (2000), pp. 51-64.

[6] Matsuyama, Y., On totally real submanifolds of a complex projective space, Nihonkai Math. J. 13 (2002), pp. 153-157.

[ 7 ] Montiel, S., Ros, A. and Urbano, F., Curvature pinching and eigenvalue rigidity for minimal submanifolds, Math. Z. 191 (1986), pp. 537-548.

[8] Nakagawa, H. and Takagi, R., On locally symmetric Kaehler submanifolds in a complex projective space, J. Math. Soc. Japan 28 (1976), pp. 638-667.

[9] Ogiue, K., Differential geometry of Kaehler submanifolds, Adv. Math. 13 (1974), pp. 73-114.

[10] Ros, A., A characterization of seven compact Kaehler submanifolds by holomorphic pinching, Ann. of Math. 31 (1981), pp. 451-454.

[11] Ros, A., Positively curved Kaehler submanifolds, Proc. Amer. Math. Soc. 93 (1985), pp. 329-331.

[12] Ros, A. and Vestraelen, V., On a conjecture of K. Ogiue, J. Differential Geom. 19 (1984), pp. 561-566.

[13] Suh, Y. J. and Yang, H. Y., Kaehler submanifolds with Ricci curvature bounded from below, Michigan Math. J. 53 (2005), pp. 545-552.

[14] Shen, Y. B., On compact Kaehler submanifolds in $C P^{n+p}$ with nonnegative sectional curvature, Proc. Amer. Math. Soc. 123 (1985), pp. 3507-3512.

[15] Tanno, S., Compact complex submanifolds immersed in complex projective space, preprint.

[16] Tsukada, K., Parallel Kaehler submanifolds of Hermitian symmetric spaces, Math. Z. 190 (1985), pp. 129-150.

[17] Umehara, M., Kaehler submanifolds of complex space forms, Tokyo J. Math. 10 (1987), pp. $203-214$.

\author{
Department of Mathematics \\ Chuo University \\ 1-13-27 Kasuga \\ Bunkyo-ku, Tokyo 112-8551, Japan \\ e-mail address: matuyama@math.chuo-u.ac.jp
}

